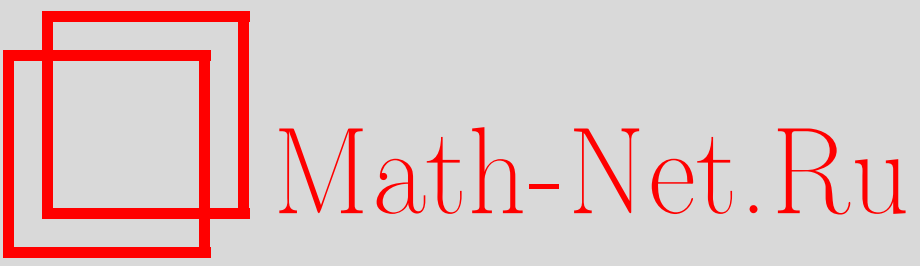

С. А. Вахрамеев, Теорема существования для нелинейной задачи быстродействия в классе релейных управлений с конечным числом переключений, $У М H$, 1996, том 51, выпуск 2, 151-152

DOI: https://doi.org/10.4213/rm951

Использование Общероссийского математического портала Math-Net.Ru подразумевает, что вы прочитали и согласны с пользовательским соглашением

http: //www . mathnet.ru/rus/agreement

Параметры загрузки:

IP : 54.174 .149 .18

26 апреля 2023 г., 14:17:12 


\title{
ТЕОРЕМА СУЩЕСТВОВАНИЯ ДЛЯ НЕЛИНЕЙНОЙ ЗАДАЧИ БЫСТРОДЕЙСТВИЯ В КЛАССЕ РЕЛЕЙНЫХ УПРАВЛЕНИЙ С КОНЕЧНЫМ ЧИСЛОМ ПЕРЕКЛЮЧЕНИЙ
}

\author{
C. A. BaxpameeB
}

Для двухточечной задачи быстродействия, связанной с гладкой нелинейной управляемой системой, устанавливается существование релейного оптимального управления без каких-либо априорных предположений о выпуклости векторграммы системы, однако при достаточно жестких условиях на структуру алгебры Ли векторных полей, ассоциированных с рассматриваемой системой.

1. Всюду далее используется язык хронологического исчисления, развитый в работах А. А. Аграчева и Р. В. Гамкрелидзе (см. [1], а также [3]). Пусть $M-n$-мерное гладкое (класса $C^{\infty}$ ) многообразие, регулярно вложенное в некоторое евклидово пространство. Рассмотрим гладкую управляемую систему

$$
\dot{x}=x \circ f(u), \quad x \in M, \quad u \in U,
$$

где $f(u), u \in \mathbb{R}^{m},-$ семейство гладких (класса $C^{\infty}$ ) векторных полей на $M$, гладко зависящих от параметра $u \in \mathbb{R}, U$ - выпуклый компактный многогранник в $\mathbb{R}^{m}$. Будем считать, что выполнено следующее условие роста: существует функция $g: \mathbb{R}_{+} \rightarrow \mathbb{R}_{+}$такая, что

$$
|x \circ f(u)| \leqslant g(|x|)
$$

для всех $x \in M, u \in U$ и

$$
\limsup _{s \rightarrow \infty} \frac{g(s)}{s}<\infty
$$

При этом условии для любого допустимого, т.е. измеримого и ограниченного управления $u(t), t \in \mathbb{R}$, со значениями в $U$, существует поток

$$
p_{t}(u)=\overrightarrow{\exp } \int_{0}^{t} f(u(s)) d s, \quad t \in \mathbb{R},
$$

на многообразии $M$.

Рассмотрим следуюшую задачу бъстродействия для (1.1):

Пусть заданы точки $x_{0}$ и $x_{T}$, требуется найти допустимое управление $u(t), t \in \mathbb{R}$, такое, что траектория

$$
x(t)=x\left(t ; x_{0}, u(\cdot)\right)=x_{0} \circ \overrightarrow{\exp } \int_{0}^{t} f(u(s)) d s=x_{0} \circ p_{t}(u), \quad t \in \mathbb{R},
$$

системь (1.1), соответствующая этому управлению и начальному состоянию $x_{0}$ $\left(x(0)=x_{0}\right)$, удовлетворяет условию $x(T)=x_{T}$ и время $T$ - наименьшее возможное.

Классическая теорема существования (см. [2]) гарантирует существование оптимального управления в этой задаче при дополнительном условии выпуклости векторграммы

$$
F(x)=\{x \circ f(u): u \in U\}
$$

для всех $x \in M$, или, более общо, при условии, что множество $F(x)$ содержит границу своей выпуклой оболочки для всех $x \in M$ (см. [2]). В случае нарушения этого условия оптимальное управление существует в классе обобщенных управлений $\mu_{t}, t \in \mathbb{R},-$ слабо измеримых семейств вероятностных мер Радона, сосредоточенных при почти всех $t \in \mathbb{R}$ на $U$. Пожалуй, единственным известным автору исключением является случай линейных по фазовой переменной систем - в этом случае теорема существования справедлива в классе "обычных" управлений. Основная цель данной заметки показать, что, по-сушеству, те же условия, что и условия релейности, предложенные автором в [5], обеспечивают существование и релейность оптимального управления без условий выпуклости векторграммы системы. 
2. Будем говорить, что система (1.1) удовлетворяет обобщенному условию общности положения (относительно многогранника $U$ ), если для любых двух вершин $e^{\prime}, e^{\prime \prime}$ этого многогранника существует $u \in U$ такое, что векторы

$$
x \circ \frac{\partial f(u)}{\partial u}\left(e^{\prime}-e^{\prime \prime}\right), x \circ \text { ad } f(u) \frac{\partial f(u)}{\partial u}\left(e^{\prime}-e^{\prime \prime}\right), \ldots, x \circ \operatorname{ad} f(u)^{n-1} \frac{\partial f(u)}{\partial u}\left(e^{\prime}-e^{\prime \prime}\right)
$$

линейно независимы при всех $x \in M$.

Будем говорить, что система (1.1) удовлетворяет обобщенному усиленному условию релейности (относительно многогранника $U$ ), если для любых вершин $e^{\prime}, e^{\prime \prime}$ многогранника $U$ и для любого компакта $K \subset M$ существуют линейные функционалы

$$
a\left(x, u^{\prime}, \cdot\right): \mathbb{R}^{m} \rightarrow \mathbb{R}, \quad b_{\beta}^{\alpha}\left(x, u^{\prime}, u^{\prime \prime}, \cdot\right): \mathbb{R}^{m} \rightarrow \mathbb{R}
$$

гладко зависящие от $(x, u) \in K \times U,\left(x, u^{\prime}, u^{\prime \prime}\right) \in K \times U \times U$, такие, что

$$
\begin{aligned}
x \circ \frac{\partial^{2} f(u)}{\partial^{2} u}(u, w) & =x \circ a(u, v) \frac{\partial f(u)}{\partial u} w \\
x \circ\left[\frac{\partial f\left(u^{\prime \prime}\right)}{\partial u} v, \operatorname{ad}^{\alpha} f\left(u^{\prime}\right) \frac{\partial f\left(u^{\prime}\right)}{\partial u}\right] & =\sum_{\beta=0}^{\alpha} x \circ b_{\beta}^{\alpha}\left(u^{\prime}, u^{\prime \prime}, v\right) \operatorname{ad}^{\beta} f\left(u^{\prime}\right) \frac{\partial f\left(u^{\prime}\right)}{\partial u} w
\end{aligned}
$$

для всех $v \in \mathbb{R}^{m}, \alpha=0,1, \ldots,(x, u) \in K \times U,\left(x, u^{\prime}, u^{\prime \prime}\right) \in K \times U \times U$, где $w=e^{\prime}-e^{\prime \prime}$.

Из (2.2), (2.3) следует, что векторы (2.1) линейно независимы для всех $u \in U$ и $x \in M$ (и для $w=e^{\prime}-e^{\prime \prime}$, где $e^{\prime}, e^{\prime \prime}$ - любые вершины $\left.U\right)$. Поэтому обобщенное условие общности положения и обобщенные усиленные условия релейности несколько сильнее соответствующих условий, предложенных автором в [5].

Основной результат данной заметки формулируется следующим образом.

Теорема. Пусть гладкая управляемая система (1.1) удовлетворяет условию роста (1.2), обобщенному условию общности положения и обобщенным усиленным условиям релейности (2.2), (2.3) относительно многогранника $U$. Тогда, если существует допустимое управление, переводящее начальное состояние $x_{0}$ в $x_{T}$, то существует и оптимальное управление $u(t), 0 \leqslant t \leqslant T$. Более того, всякое оптимальное управление кусочно постоянно и его значениями являются только вериины многогранника $U$.

\section{СПИСОК ЛИТЕРАТУРЫ}

[1] Аграчев А. А., Гамкрелидзе Р. В. // Матем. сб. 1978. Т. 100. №4. С. 467-532. [2] Гамкрелидзе Р. В. Основы оптимального управления. Тбилиси: Изд-во Тбилисского ун-та, 1975. [3] Гамкрелидзе Р. В., Аграчев А. А., Вахрамеев С. А. // Итоги науки и техники. Соврем. пробл. матем. Новейш. достиж. 1989. Т. 35. С. 3-107. [4] Вахрамеев С. А. // Итоги науки и техники. Соврем. пробл. матем. Новейш. достиж. 1989. Т. 35. С. 135-177. [5] Вахрамеев С. А. // УМН. 1994. Т. 49. № 4. С. 197-198.

Всероссийский институт научной и технической информации (ВИНИТИ) 